Nervenarzt 2010 $\cdot 81: 667-668$

DOI 10.1007/s00115-010-2942-7

Online publiziert: 27. Mai 2010

(c) Springer-Verlag 2010

\author{
G. Deuschl \\ Klinik für Neurologie, Universitätsklinikum Schleswig-Holstein, Campus Kiel
}

\section{Tiefe Hirnstimulation}

\section{Höhere Lebensqualität mit Strom als mit Medikamenten}

Die tiefe Hirnstimulation (THS) ist für die Parkinson-Erkrankung der größte Behandlungsfortschritt seit der Entdeckung des L-Dopa in den 6oer Jahren des vergangenen Jahrhunderts. Gleichermaßen ist sie auch der entscheidende Fortschritt in der Behandlung zahlreicher schwerer und behandlungsresistenter Tremores und Dystonien. Sehr häufig wird den oft schwerstbehinderten Patienten eine Rückkehr in ein Leben mit geringer oder doch zumindest akzeptabler Behinderung ermöglicht.

Obwohl am Beginn der heute so erfolgreichen Stimulation des Nucleus subthalamicus für die Parkinson-Krankheit die Aufklärung der Basalganglienschleife stand, war die Entwicklung der Behandlung insgesamt keineswegs durch systematische Grundlagenforschung vorangetrieben worden. Sie basiert für alle Zielorte maßgeblich auf den Erfahrungen der läsionellen stereotaktischen Chirurgie der 2. Hälfte des 20. Jahrhunderts. Der entscheidende Unterschied zu dieser Behandlung ist aber, dass mit der THS plötzlich ein weitgehend reversibles Verfahren zur Verfügung steht. Bis heute gilt als ärztlicher Grundsatz, dass die Therapie so wenig invasiv wie möglich bleiben sollte. Dass Neurologen und Psychiater die THS zunehmend erwägen, obwohl sie eigentlich die konservativen „Ärzte des Gehirns“ sind, ist einzig auf die oft spektakulären klinischen Erfolge zurückzuführen. Nebenwirkungen und Operationsrisiken müssen aber sorgfältigst abgewogen werden. Sie sind jedoch heute sehr genau kalkulierbar und für die großen Indikationen gut bekannt. Dies wird im Beitrag von J. Voges und J. Krauss detailliert erläutert.

Das vorliegende Themenschwerpunktheft gibt einen Einblick in die für die gesamte Neurologie und Psychiatrie wichtige Entwicklung der THS, die erst am Beginn steht. Dabei werden zwei Ziele verfolgt: Erstens soll dieses Heft über die heute schon in praktische Therapie umgesetzten Möglichkeiten informieren. Zweitens soll ein Einblick in die Forschung gegeben werden, wie zukünftig neue Einsatzgebiete gefunden werden können und wie durch systematische Forschung Wirkmechanismen aufgeklärt werden und der neurochirurgische Eingriff noch präziser und sicherer gemacht werden kann.

\section{( Die THS ist bei fortgeschrittenem \\ M. Parkinson auf höchstem Evidenzniveau wirksam}

Das Themenheft beginnt mit den heute schon etablierten Indikationen. Für die Parkinson-Erkrankung liegen mittlerweile die Erfahrungen von mehr als 30.00o Behandlungen weltweit vor. Viel wichtiger aber als große Zahlen sind die kontrollierten Studien, die uns den Vergleich mit den etablierten Behandlungen erlauben. Die THS ist danach auf höchstem Evidenzniveau bei fortgeschrittenem M. Parkinson mit medikamentös nicht mehr behandelbaren Fluktuationen wirksamer als die medikamentöse Behandlung (Beitrag J. Herzog, G. Deuschl). Vergleichbar gute Evidenz liegt für die Behandlung der Dystonien vor. Allerdings ist die Patientenzahl hier viel kleiner. Beim Tremor sind die Erfolge ebenfalls groß, aber systematische kontrollierte Studien sind hier eine Rarität (Beitrag L. Timmermann, J. Volkmann). Beide Artikel fokussieren auf die Behandlungserfolge und den Abwägungsprozess bei der Indikationsstellung. Die Langzeitbegleitung solcher Patienten ist mittlerweile ein wichtiges Thema auch für die niedergelassenen Kollegen. Deshalb werden die medizinischen und psychologischen Anforderungen im Prozess der kontinuierlichen Begleitung dieser Patienten in einem separaten Artikel adressiert (Beitrag F. Sixel-Döring, G. Ebersbach). Im Gegensatz zu diesen etablierten Indikationen werden die psychiatrischen Erkrankungen, Zwangserkrankungen, Depression und Sucht bislang nur in klinischen Studien erprobt (Beitrag T. Schläpfer, S. Kayser). Auch hier deutet sich jedoch schon an, dass zumindest bei einigen Erkrankungen große Therapieerfolge möglich scheinen.

Diese großen klinischen Erfolge haben uns gelehrt, dass mit der gezielten elektrischen Stimulation umschriebener Hirnareale für einige Krankheiten ein großer Fortschritt erzielt werden kann. Es liegt nahe anzunehmen, dass dies noch für weitere Hirnkrankheiten gilt. Der Neurologe denkt an schwerste idiopathische Kopfschmerzen, Epilepsien, die Ataxie vielleicht sogar bis zu einem gewissen Grade an die Demenz. Der Psychiater denkt an die Depression, die Schizophrenie und Zwangs- und Suchtkrankheiten. Natürlich muss dazu zunächst verstanden werden, welche funktionellen Veränderungen bei diesen Krankheiten in der Kooperation verschiedener Hirnareale 
vorliegen. Dies muss durch gezielte Forschung in verschiedener Richtung vorbereitet werden. Ein Aspekt ist dabei die Frage nach dem zellulären Wirkmechanismus dieser Behandlung, der immer noch nicht vollständig aufgeklärt ist. Im Beitrag von J. Voges und J. Krauss wird das heutige Wissen vorgestellt. Wenn langfristig auch andere Erkrankungen behandeln werden sollen, die mit einer veränderten Kooperation der Hirnareale einhergehen, so muss vor allem auf Systemebene geforscht werden. Es muss also untersucht werden, wie die Entladungscharakteristika umschriebener Hirnareale bei den Erkrankungen verändert sind und wie sich die Hirnaktivität durch gezielte Stimulation an verschiedenen Orten beeinflussen lässt. Dies wird nicht im Reagenzglas, sondern nur im Tierexperiment verstanden werden können und daher muss der Einsatz der THS bei verschiedenen Tiermodellen von Krankheiten untersucht werden. Im Beitrag von C. Winter, D. Harnack und A. Kupsch wird diese Forschung für einige neurologische und psychiatrische Krankheitsbilder vorgestellt. Die ethischen Implikationen dieser Therapieentwicklung sind ein wichtiger Bereich, der in einem späteren Beitrag besprochen werden wird.

Für viele Neurologen ist die Betreuung von Patienten mit THS zum Alltag geworden. Für die Psychiater wird es das vielleicht werden. Es ist aber heute schon klar, dass diese Behandlung das Spektrum der Alltagstätigkeit des Neurologen bereichern wird. Wir dürfen derzeit eine Zeit erleben in der neben die Medikamente ein Therapieprinzip tritt, das völlig anders funktioniert und das uns neue therapeutische Möglichkeiten gibt.

Die Stimulationstherapie, von denen die Nervenärzte seit Jahrhunderten träumten, ist heute für einige Krankheiten Realität geworden. Eine weitergreifende Perspektive für zahlreiche andere Erkrankungen erwartet uns. Der Weg dorthin muss über eine systemorientierte Grundlagenforschung im Tier und eine Krankheitsforschung mit nichtinvasiven systemischen Methoden wie etwa die funktionelle Bildgebung oder EEG/MEG-Untersuchungen gehen. Sollte dann ein neuer Stimulationsort für eine Krankheit gefunden werden, so bedarf es sorgfältiger kontrollierter klinischer Studien, um deren therapeutisches Potenzial beim Kranken abzuschätzen. So wird eine neue Therapierichtung entwickelt werden, die für die Generationen nach uns wahrscheinlich völlig selbstverständlich neben der medikamentösen Therapie stehen wird.

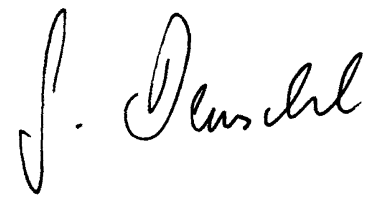

\section{G. Deuschl}

\section{Korrespondenzadresse \\ Prof. Dr. G. Deuschl}

Klinik für Neurologie, Universitätsklinikum Schleswig-Holstein, Campus Kiel Schittenhelmstraße 10, 24105 Kiel g.deuschl@neurologie.uni-kiel.de.
Wolffram-Preis für Forschungsarbeiten zum Thema Kopf-

\section{schmerz}

Ausschreibung der Deutschen Migräne- und Kopfschmerzgesellschaft (DMKG)

Eingereicht werden können Diplomarbeiten, Doktorarbeiten und Publikationen (Publikationen sollten zumindest ,im Druck" sein bzw. nicht älter als 12 Monate). Für den Preis können sich wissenschaftliche Mitarbeiterinnen und Mitarbeiter aller Fachrichtungen bewerben, die an einer akademischen Einrichtung in Deutschland tätig sind. Es besteht keine Altersbegrenzung.

Der erste Preis wird mit $5.000 €$, der zweite mit $2.500 €$ und der dritte mit $1.000 €$ gewürdigt.

Die Bewertung nimmt das DMKG-Präsidium vor. Dieses behält sich vor, externe Gutachter hinzuzuziehen.

Der Wolffram-Preis wird anlässlich des Deutschen Schmerzkongresses vom 6. - 9.10. 2010 in Mannheim verliehen. Bewerbungen sind in vierfacher Ausfertigung bis zum 15. August 2010 (Poststempel) zu richten an:

PD Dr. Stefanie Förderreuther Generalsekretärin der DMKG

Neurologische Klinik der Universität München Konsiliardienst am Standort Innenstadt Ziemssenstrasse 1 80336 München

Quelle: www.dmkg.de 